\title{
THE SUBMILLIMETER WAVE ASTRONOMY SATELLITE
}

\author{
G.J. MELNICK \\ Harvard-Smithsonian Center for Astrophysics
}

\begin{abstract}
The Submillimeter Wave Astronomy Satellite (SWAS) is a NASA SmallExplorer Class experiment whose objective is to study both the chemical composition and the thermal balance in dense $\left(\mathrm{N}_{\mathrm{H}_{2}}>10^{3} \mathrm{~cm}^{-3}\right)$ molecular clouds and, by observing many clouds throughout our galaxy, relate these conditions to the processes of star formation. To conduct this study SWAS will be capable of carrying out both pointed and scanning observations simultaneously in the lines of four important species: $(1)$ the $\mathrm{H}_{2} \mathrm{O}\left(1_{10}-1_{01}\right)$ $556.963 \mathrm{GHz}$ ground-state ortho transition, (2) the $\mathrm{O}_{2}(3,3-1,2) 487.249 \mathrm{GHz}$ transition, (3) the CI $\left({ }^{3} P_{1}-{ }^{3} P_{0}\right) 492.162 \mathrm{GHz}$ ground-state fine structure transition, and (4) the ${ }^{13} \mathrm{CO}(\mathrm{J}=5-4) 550.926 \mathrm{GHz}$ rotational transition. These atoms and molecules are predicted to be among the most abundant within molecular clouds and, because they possess low-lying transitions with energy differences $(\Delta E / k)$ between 15 and $30 \mathrm{~K}$ (temperatures typical of many molecular clouds), these species are believed to be dominant coolants of the gas as it collapses to form stars and planets. A large-scale survey in these lines is virtually impossible from any platform within the atmosphere due to telluric absorption.

SWAS will consist of a 55-cm diameter off-axis Cassegrain antenna, two Schottky Barrier diode heterodyne radiometers, and a single broadband (1.4 GHz) acousto-optical spectrometer (AOS) which will give SWAS a velocity resolution of approximately 0.6 $\mathrm{km} \mathrm{s}^{-1}$. The spacecraft, supplied by NASA Goddard Space Flight Center, will be a threeaxis all-sky pointer utilizing reaction wheels. Use of a star tracker will permit a pointing accuracy of approximately 45 arcseconds. The satellite has a weight of $200 \mathrm{~kg}$, carries no expendables, and, with a projected orbital altitude of $550-600 \mathrm{~km}$, should function for two or more years. Launch is currently scheduled for 1994. SWAS is a collaborative effort between the Smithsonian Astrophysical Observatory, the University of Massachusetts at Amherst, the National Air and Space Museum, NASA Ames Research Center, the University of California at Berkeley, and the University of Cologne (FRG).
\end{abstract}

